\title{
Mechanical CPR devices compared to manual CPR during out-of-hospital cardiac arrest and ambulance transport: a systematic review
}

Marcus Eng Hock Ong ${ }^{1 *}$, Kevin E Mackey ${ }^{2}$, Zhong Cheng Zhang ${ }^{1}$, Hideharu Tanaka ${ }^{3}$, Matthew Huei-Ming Ma ${ }^{4}$, Robert Swor ${ }^{5}$ and Sang Do Shin 6

\begin{abstract}
Aims: The aim of this paper was to conduct a systematic review of the published literature to address the question: "In pre-hospital adult cardiac arrest (asystole, pulseless electrical activity, pulseless Ventricular Tachycardia and Ventricular Fibrillation), does the use of mechanical Cardio-Pulmonary Resuscitation (CPR) devices compared to manual CPR during Out-of-Hospital Cardiac Arrest and ambulance transport, improve outcomes (e.g. Quality of CPR, Return Of Spontaneous Circulation, Survival)".

Methods: Databases including PubMed, Cochrane Library (including Cochrane database for systematic reviews and Cochrane Central Register of Controlled Trials), Embase, and AHA EndNote Master Library were systematically searched. Further references were gathered from cross-references from articles and reviews as well as forward search using SCOPUS and Google scholar. The inclusion criteria for this review included manikin and human studies of adult cardiac arrest and anti-arrhythmic agents, peer-review. Excluded were review articles, case series and case reports.

Results: Out of 88 articles identified, only 10 studies met the inclusion criteria for further review. Of these 10 articles, 1 was Level of Evidence (LOE) 1, 4 LOE 2, 3 LOE 3, 0 LOE 4, 2 LOE 5. 4 studies evaluated the quality of CPR in terms of compression adequacy while the remaining six studies evaluated on clinical outcomes in terms of return of spontaneous circulation (ROSC), survival to hospital admission, survival to discharge and Cerebral Performance Categories (CPC). 7 studies were supporting the clinical question, 1 neutral and 2 opposing.

Conclusion: In this review, we found insufficient evidence to support or refute the use of mechanical CPR devices in settings of out-of-hospital cardiac arrest and during ambulance transport. While there is some low quality evidence suggesting that mechanical CPR can improve consistency and reduce interruptions in chest compressions, there is no evidence that mechanical CPR devices improve survival, to the contrary they may worsen neurological outcome.
\end{abstract}

Keywords: "Mechanical", "Automatic", "Load distribution band, "Cardiopulmonary resuscitation", "Chest compression", "Transport", and "Transportation"

\section{Background}

Sudden cardiac arrest is a global concern. Over the years, the mechanism of death has shifted from ventricular fibrillation (VF) as initial rhythm to pulseless electrical activity (PEA) and asystole [1-4]. The incidence of out-of-hospital cardiac arrest (OHCA) in USA has been estimated at 1.89/1,000 person-years and at 5.98/1,000

\footnotetext{
* Correspondence: marcus.ong.e.h@sgh.com.sg

'Department of Emergency Medicine, Singapore General Hospital, Outram Road, Singapore 169608, Singapore

Full list of author information is available at the end of the article
}

person-years in subjects with any clinically recognized heart disease [5]. Published survival rates for OHCA ranged from $3.0 \%$ to $16.3 \%$ in North America [6].

$\mathrm{CPR}$ during OHCA and ambulance transport is a key issue in pre-hospital emergency care. CPR in a moving ambulance is a particular challenge. Patients may arrest during transport, or OHCA patients may need to be transported due to local ambulance policies. For example, in Asia-Pacific countries, societal and social norms see pronouncement of death in residences and public places as taboo. Thus, cardiac arrest patients are

\section{Biomed Central}


often transported to the hospital in a 'scoop and run' model. This is in contrast to the practice in North American Emergency Medical Services (EMS) systems and European communities, where CPR is more often performed at scene, and unsuccessful resuscitations may be terminated in the field.

The quality of CPR during OHCA is a key factor affecting survival [7]. The problem with standard CPR is that it provides only one third of normal blood supply to the brain and 10-20\% of normal blood flow to the heart [8]. Good quality CPR is an even greater challenge in the setting of a moving ambulance. It is also increasingly recognized that although defibrillation is the definitive treatment for ventricular fibrillation, its success is also dependent on adequate circulation [9-11]. Thus, effective CPR is often a prerequisite for effective defibrillation.

Mechanical CPR is an attractive alternative, due to theoretical advantages including elimination of the rescuer fatigue factor, more consistent and reliable chest compression, and eliminating the need to stop CPR during rescuer changes and patient transfers. It is also conceivably safer for the ambulance crew during transport. However results from clinical trials have been conflicting, with studies suggesting a benefit for mechanical CPR [12] and others that failed to find any significant difference between manual and mechanical CPR in survival to discharge [13]. Possible explanations for these unexpected results advanced by the authors included a Hawthorne effect, prolonged deployment time for the devices resulting in delayed defibrillation and enrollment bias [13].

The aim of this paper was to conduct a systematic review of the published literature on the use of mechanical CPR devices compared to manual CPR during OHCA and ambulance transport in pre-hospital adult cardiac arrest regardless of presenting rhythm. This review was conducted as part of a consensus meeting on ambulance CPR, organized by the Asian EMS Council in 2011 (Seoul, Korea). More information about the council can be found at: http://www.scri.edu.sg/index.php/parosclinical-research-network.

\section{Methods}

The review was conducted in accordance with the methodology recommended by the International Liaison Committee on Resuscitation (ILCOR) 2010 evidence evaluation process [14] where this sought to identify evidence to address the question: [15] "In pre-hospital adult cardiac arrest (asystole, pulseless electrical activity, pulseless VT and VF)(pre-hospital [OHCA]), does the use of mechanical CPR devices compared to manual CPR during ambulance transport, improve outcomes (e.g. quality of CPR, return of spontaneous circulation (ROSC), survival)".
A search strategy was pursued, using the following search terms: "mechanical", "automatic", "load distribution band", "cardiopulmonary resuscitation", "chest compression", "transport" and "transportation" (textword and $\mathrm{MeSH}$ headings when applicable).

Databases were searched up to 1 October 2011, with PubMed, the Cochrane Library (including Cochrane database for systematic reviews and Cochrane Central Register of Controlled Trials), Embase, and the American Heart Association (AHA) Resuscitation Endnote Master library, which contains over 15,000 cardiac arrest related references. Moreover, cross-references from articles and reviews were forward searched using SCOPUS and Google scholar.

In addition, we looked at the following review articles: a) Manual versus Mechanical Chest compression for cardiac arrest (Cochrane Review) [16], b) CPR techniques and devices: 2010 International Consensus on Cardiopulmonary Resuscitation and Emergency Cardiovascular Care Science with Treatment Recommendation (AHA) [17]. Cross references from these review articles were forward searched using SCOPUS and Google Scholar.

Inclusion criteria were human studies of adult cardiac arrest (age $\geq 16$ ) and manikin studies which were peerreviewed. Exclusion criteria were case reports and case series. We broadened the scope of our review from CPR solely during ambulance transport to OHCA in general due to the absence of clinical outcome studies addressing this particular setting.

The articles were reviewed for relevance independently by two reviewers $(\mathrm{MEHO} / \mathrm{KM})$. Both titles and abstracts were reviewed, followed by the articles if suitable for review. Articles where the content was clearly unrelated were discarded. The abstracts of remaining articles were then reviewed and relevant studies identified for detailed review of the full manuscript. Where disagreement existed between reviewers, articles were included for detailed review. Finally, the reference lists of narrative reviews were examined to identify any additional articles not captured by the main search strategy.

\section{Evidence appraisal}

Studies were reviewed in detail and then classified by level of evidence (LOE) (Table 1) and quality (rated poor, fair or good) according to agreed definitions (Table 2). "Methodological quality" (internal validity) of a study was defined as "the extent to which a study's design, conduct, and analysis has minimized selection, measurement, and confounding biases" [14]. That quality is separate to "non-methodological" quality, which refers to the external validity or generalizability of the study results to other (broader) population groups.

Studies were allocated a rating for methodological quality (Good, Fair or Poor) according to the presence of 
Table 1 International Liaison Committee on Resuscitation (ILCOR) 2010 Levels of Evidence for Studies of Therapeutic Interventions

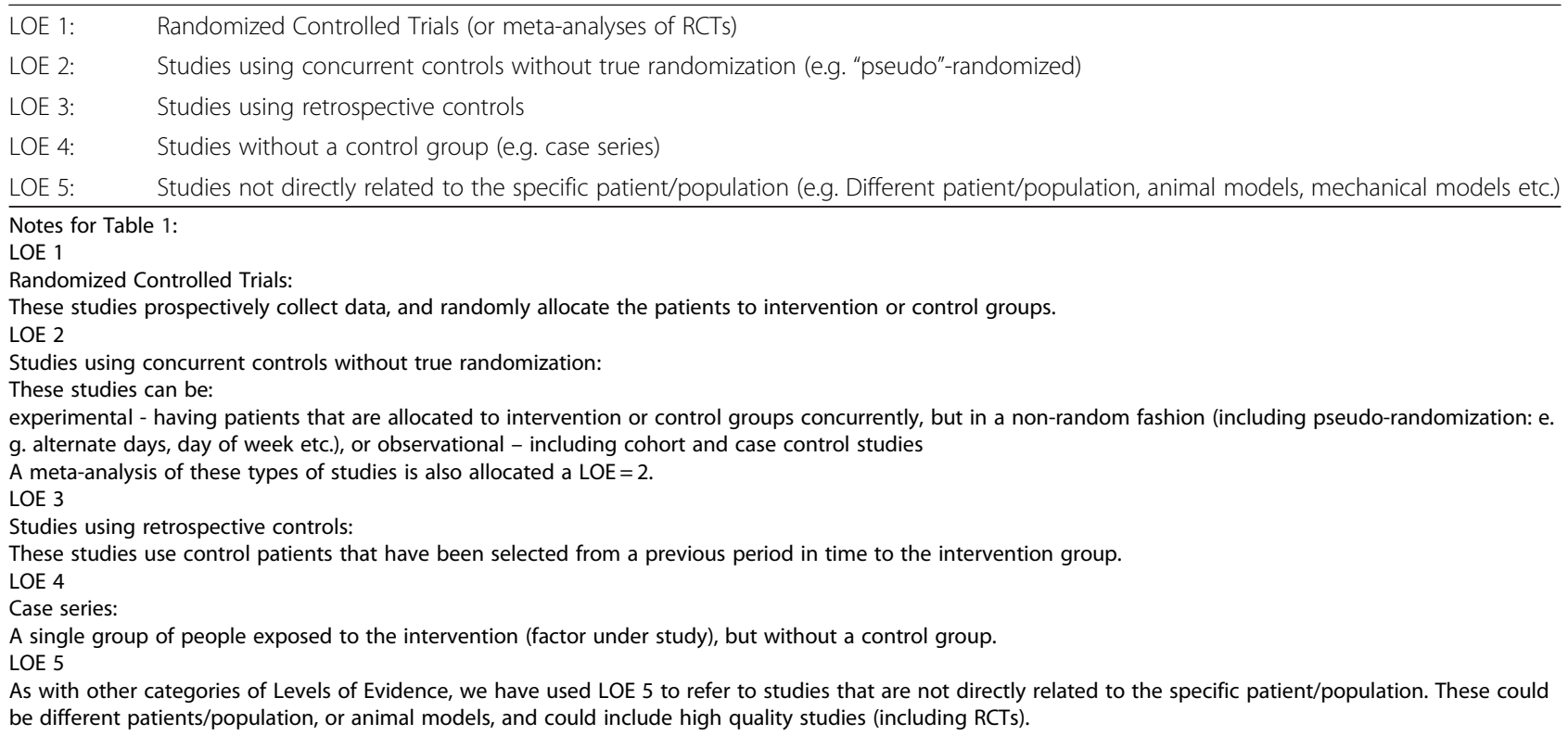

the quality items for that Level of Evidence (see Table 2). Good studies had most/all of the relevant quality items, Fair studies had some of the relevant quality items and Poor studies had few of the relevant quality items (but sufficient value to include for further review).

\section{Results}

Out of 88 articles identified, 10 studies met the inclusion criteria for further review. Of these 10 articles, 1 was LOE 1, 4 LOE 2, 3 LOE 3, 0 LOE 4, 2 LOE 5 (see Table 3).

Table 4 is a summary of our review of all eligible studies.

\section{Studies comparing quality of CPR}

Two manikin studies comparing CPR quality suggested that mechanical CPR performs better during ambulance transport. In the prospective and observational study $(\mathrm{N}=144)$ conducted by Stapleton [18], mechanical CPR was able to provide correct compressions for overall $97 \%$ of the runs as compared to $37 \%$ of the runs in manual CPR. The 97\% rate remained valid for different environmental settings such as vehicle type and road conditions while manual compressions varied upon different settings. Similarly, Sunde [19] noted that compression depth and frequency provided by mechanical CPR devices were better at both cardiac arrest sites and during transport as they were within CPR standards. An increase in weak compressions for manual CPR was observed during ambulance transport. The recommended compression-decompression ratio of 50/50 was only achieved with the device as compared to other techniques where the ratio was significantly lower.

Similar observations were made by Olasveengen [22] where a retrospective and observational study was conducted to evaluate the quality of CPR before and during transport on adult patients with OHCA. In both settings, hands-off ratio for mechanical CPR was shorter than manual CPR and chest compression rates for mechanical $\mathrm{CPR}$ were closer to recommended guidelines. Cerebral Performance Categories (CPC) scores were also gathered but no conclusions could be drawn to patient's outcome due to overall small sample size $(\mathrm{N}=75)$.

However, conflicting results were shown in a prospective and randomized study conducted by Wang [25]. The average chest compression rate and ventilation rate was significantly lower (related to the setting) for mechanical CPR ( $<<0.05, \mathrm{p}<0.05)$ although there was lesser variability in the instantaneous chest compression rate. Due to short transport time of average 4 minutes and small sample size $(\mathrm{N}=20)$, the study indicated no significant difference in average no-chest compression interval.

\section{Studies comparing clinical outcomes}

Many studies did not separate patients who required $\mathrm{CPR}$ at scene from those who required it during transport. However, most articles indicated that the patients were loaded with the CPR device onto the ambulance and then transported with CPR ongoing.

A retrospective case-control study $(\mathrm{N}=262)$ conducted by Casner [23] found that for mechanical CPR, higher rates of ROSC was observed in patients overall 
Table 2 Quality assessment for studies assessing interventions

\begin{tabular}{|c|c|}
\hline \multirow[t]{17}{*}{ LOE 1} & Quality assessment for Randomized Controlled Trials \\
\hline & The seven factors included as the relevant quality items for RCTs are: \\
\hline & - Was the assignment of patients to treatment randomized? \\
\hline & - Was the randomization list concealed? \\
\hline & - Were all patients who entered the trial accounted for at its conclusion? \\
\hline & - Were the patients analyzed in the groups to which they were randomized? \\
\hline & . Were patients and clinicians "blinded" to which treatment was being received? \\
\hline & - Aside from the experimental treatment, were the groups treated equally? \\
\hline & - Were the groups similar at the start of the trial? \\
\hline & Quality assessment for meta-analyses of RCTs \\
\hline & The six factors included as the relevant quality items for meta-analyses are: \\
\hline & $\begin{array}{l}\text { - Were specific objectives of the review stated (based on a specific clinical question in which patient, } \\
\text { intervention, comparator, outcome (PICO) were specified) }\end{array}$ \\
\hline & . Was study design defined? \\
\hline & -Were selection criteria stated for studies to be included (based on trial design and methodological quality)? \\
\hline & -Were inclusive searches undertaken (using appropriately crafted search strategies)? \\
\hline & - Were characteristics and methodological quality of each trial identified? \\
\hline & -Were selection criteria applied and a log of excluded studies with reasons for exclusion reported? \\
\hline \multirow[t]{14}{*}{ LOE 2} & Quality assessment for studies using concurrent controls without true randomization \\
\hline & The four factors included as the relevant quality items for these studies are: \\
\hline & -Were comparison groups clearly defined? \\
\hline & -Were outcomes measured in the same (preferably blinded), objective way in both groups? \\
\hline & - Were known confounders identified and appropriately controlled for? \\
\hline & - Was follow-up of patients sufficiently long and complete? \\
\hline & Quality assessment for meta-analyses of studies using concurrent controls without true randomization \\
\hline & The six factors included as the relevant quality items for meta-analyses are: \\
\hline & $\begin{array}{l}\text { - Were specific objectives of the review stated (based on a specific clinical question in which patient, intervention, } \\
\text { comparator, outcome (PICO) were specified) }\end{array}$ \\
\hline & - Was study design defined? \\
\hline & -Were selection criteria stated for studies to be included (based on trial design and methodological quality)? \\
\hline & -Were inclusive searches undertaken (using appropriately crafted search strategies)? \\
\hline & - Were characteristics and methodological quality of each trial identified? \\
\hline & - Were selection criteria applied and a log of excluded studies with reasons for exclusion reported? \\
\hline \multirow[t]{6}{*}{ LOE 3} & Quality assessment for studies using retrospective controls: \\
\hline & The four factors included as the relevant quality items for these studies are: \\
\hline & - Were comparison groups clearly defined? \\
\hline & -Were outcomes measured in the same (preferably blinded), objective way in both groups? \\
\hline & - Were known confounders identified and appropriately controlled for? \\
\hline & - Was follow-up of patients sufficiently long and complete? \\
\hline \multirow[t]{5}{*}{ LOE 4} & Quality assessment for case series \\
\hline & The three factors included as the relevant quality items for these studies are: \\
\hline & - Were outcomes measured in an objective way? \\
\hline & - Were known confounders identified and appropriately controlled for? \\
\hline & - Was follow-up of patients sufficiently long and complete? \\
\hline \multirow[t]{5}{*}{ LOE 5} & Quality assessment for studies that are not directly related to the specific patient/population \\
\hline & $\begin{array}{l}\text { LOE } 5 \text { studies are those not directly related to the specific patient/population (e.g. different patient/population, } \\
\text { animal models, mechanical models etc.), and should have their methodological quality allocated to the } \\
\text { methodology of the study. The relevant quality criteria here are: }\end{array}$ \\
\hline & - Good = randomized controlled trials (equivalent of LOE 1) \\
\hline & - Fair = studies without randomized controls (equivalent of LOE 2-3) \\
\hline & . Poor = studies without controls (equivalent of LOE 4). \\
\hline
\end{tabular}


Table 3 Summary of evidence

\begin{tabular}{|c|c|c|c|c|c|}
\hline Level of Evidence & 1 & 2 & 3 & 4 & 5 \\
\hline \multicolumn{6}{|c|}{ Evidence SUPPORTING Clinical Question } \\
\hline Good & & & & & Stapleton [18], Sunde [19] \\
\hline Fair & & Axelsson [20], Dickinson [21] & Olasveengen [22], Ong [12], Casner [23] & & \\
\hline \multicolumn{6}{|l|}{ Poor } \\
\hline \multicolumn{6}{|c|}{ Evidence NEUTRAL Clinical Question } \\
\hline \multicolumn{6}{|l|}{ Good } \\
\hline \multicolumn{6}{|l|}{ Fair } \\
\hline Poor & & Axelsson [24] & & & \\
\hline \multicolumn{6}{|c|}{ Evidence OPPOSING Clinical Question } \\
\hline Good & Hallstrom [13] & & & & \\
\hline Fair & & Wang [25] & & & \\
\hline Poor & & & & & \\
\hline
\end{tabular}

(39\% vs. $29 \%, \mathrm{p}<0.05)$ and in asytole (37\% vs. $22 \%$, p $<0.05$ ) upon arrival at the Emergency department. There was no significant difference in ROSC for subgroups with shockable rhythms and PEA. However, these results might be confounded by selective late deployment of the mechanical CPR device in patients not responding to resuscitation.

Ong [12] compared both types of CPR in a phased, nonrandomized, observational study $(\mathrm{N}=783)$ of clinical outcomes of patients treated before and after transition from manual CPR to mechanical CPR. The use of mechanical CPR had higher rates of ROSC (34.5\% vs. $20.2 \%, \mathrm{p}<0.05)$ and survival to hospital admission $(20.9 \%$ vs. $11.1 \%$, p $<0.05)$. For survival to hospital discharge, it was better than manual CPR and there was no significant difference in CPC categories $(\mathrm{p}=0.36)$ and Overall Performance Categories $(\mathrm{p}=0.40)$ between both groups.

However, results obtained from Ong [12] were different from a randomized trial $(\mathrm{N}=1071)$ conducted by Hallstrom [13] where the same mechanical CPR device used resulted in worse neurological outcomes and worse survival. Hallstrom's study found that there was no significant difference between both types of CPR for return of spontaneous circulation 4 hours after the call to 911 was made $(29.5 \%$ vs. $28.5 \%, \mathrm{p}=0.74)$. For mechanical CPR, survival to hospital discharge was poorer (5.8\% vs. 9.9\%, $\mathrm{p}=0.06$ ) and CPC score of 1 or 2 was smaller as compared to manual CPR (3.1\% vs. $7.5 \%$, p <0.05).

A similar study conducted by Axelsson [24] showed that there was no significant difference between both types of CPR with regards to ROSC, survival to hospital discharge or to hospital discharge. No difference between CPC score was also observed. Axelsson [20] also evaluated the cardiac output between both types of CPR, measured by pressure of end-tidal carbon dioxide $\left(\mathrm{ETCO}_{2}\right)$. He found that mechanical CPR resulted in higher $\mathrm{ETCO}_{2}$ as compared to manual CPR in terms of average values $(3.26$ vs. $2.69, \mathrm{p}<0.05)$, initial value $(3.38$ vs. $2.71, \mathrm{p}<0.05)$ and minimum value (2.24 vs. $1.69, \mathrm{p}$ $<0.05)$. No significant differences were observed in maximum value ( 4.88 vs. $4.48, \mathrm{p}=0.23$ ).

These observations were similarly found in the study initiated by Dickinson [21] who compared whether a mechanical device is better in providing CPR than manual $\mathrm{CPR}$ as measured by $\mathrm{ETCO}_{2}$ and whether it is capable of improving survival outcomes. In the prospective, odd/even day randomized study conducted $(\mathrm{N}=20)$, he identified that there is sustained or increased $\mathrm{ETCO}_{2}$ with the use of mechanical CPR as compared to manual CPR. No difference in patient outcomes was observed between both groups as the study conducted was too small to draw any solid conclusion between two study groups.

\section{Discussion}

Cardiac arrest offers several challenges to the provider when he or she is working to achieve return of spontaneous circulation. Preliminary data suggests that the maintenance of quality CPR declines the moment a patient is moved toward the ambulance or transported to the hospital [26]. Variability in both the depth and the rate of compressions increases dramatically translating to a decline in CPR quality. Beginning from the first patient movement, the provider is often forced to negotiate tight doorways, narrow stairwells, uneven pavement or gravel or dirt just to get the patient to the ambulance. Transporting the patient presents a whole different set of challenges including the rocking of the ambulance, sudden stops, accelerations, decelerations and turns, roadway conditions, not to mention the physical layout of the passenger compartment of the ambulance and how many other providers are present rendering care. Each of these factors contributes in some way to reducing the quality of CPR being delivered. In this paper, a comprehensive review of studies to date relating to the quality of $\mathrm{CPR}$ and clinical outcomes when comparing manual to mechanical CPR in OHCA was evaluated. 
Table 4 Review of eligible studies

\section{S/N Title}

1 Comparing CPR

ambulance transport:

Manual versus Mechanical

methods [18]

\section{Description of Subjects Study Design}

Prospective observational

144 simulated runs

conducted on

manikin

Prospective observational
study comparing between

both types of CPR in

different environment

settings such as vehicle

type, road conditions,

vehicle speed.

Thumper was used.

2 Quality of mechanical, manual standard and active compression-decompression CPR on the arrest site and during transport in a manikin model [19]

3 Quality of cardiopulmonary resuscitation before and during transport in out-of hospital cardiac arrest [22]

$4 \quad$ Video-recording and time motion analysis of manua versus mechanical cardiopulmonary

resuscitation during ambulance transport [25]

36 runs conducted on a Randomized, cross-over, manikin which runs are out-of-hospital study

split between 3 groups. comparing between three type of CPR at cardiac arrests sites and during transportation.

Adult patients over 18 Retrospective years of age suffering observational study. from non-traumatic LUCAS was used.

OHCA for all causes and received CPR before and during transportation.

Manual CPR during

transport: 66

Mechanical CPR during

transport: 9

Adult patients over

18 years of age with

OHCA for all rhythms.

Manual: 12

Mechanical: 8 recording and time-
5 The impact of a New CPR Adult patients with Assist Device on rate of return OHCA. No exclusions of spontaneous Circulation in listed. Out of hospital Cardiac Arrest [23]

Manual: 138

Mechanical: 124

\section{Outcome}

Compression adequacy

(defined as 1.5 to 2 inches

in depth, 60 compressions

per min $+/-10 \%$ and

correct hand position).

\section{Prospective and randomized} study where videomotion studies was conducted.

Thumper was used.

\section{Results}

Thumper provided pre-defined

correct compressions for $97 \%$

of the runs $(n=70)$ while manual

compressions were performed

correctly $37 \%$ of the runs $(n=27)$.

$97 \%$ rate for the device remains

valid for different environment

settings while $37 \%$ rate for manua

CPR varied in different settings.

Compression depth, frequency, Compression depth, frequency

compression - decompression and compression ratio in mechanical closely to ERC guidelines. Working $\begin{array}{lll}\text { ratio based on European } & \text { CPR device were within } & \text { conditions for standard CPR } \\ \text { Resuscitation Council } & \text { recommended guidelines during } & \text { in ambulance transport is }\end{array}$

Resuscitation Counci

Guidelines (ERC).

recommended guidelines during

ambulance transports as compared to other 2 groups.

Chest compression and ventilation rates, hands-off mechanical CPR both before ratio (time without compressions divided by total (PR time). mechanical CPR both before
$(p=0.016)$ and during transport $(p=0.016)$
$(p=0.002)$.

Compression rates for mechanical CPR were closer to recommended guidelines (100/min) while manual CPR was higher (96/min vs. 119/min) undesirable.

For manual CPR, Time without chest compressions during transport and compression rate was lower due to difficult conditions. As such, the device is more appropriate. No conclusion is drawn about patient outcomes due to small sample size.

No difference in CPC scores

between two groups.

No-chest compression interval (Mechanical: $33.4 \%$ vs. Manual: (n) rates, unnecessary no chest compression interval, based on guidelines in ILCOR.

Average chest compression rate excluding no chest compression interval (mechanical: $52.3 / \mathrm{min}$ vs. manual: $113.3 / \mathrm{min}, \mathrm{p}<0.050$ )

Lower ventilation rate excluding no chest compression interval (mechanical 11.7/min vs. manual $16.1 /$ min, $p<0.050$ ).

Higher variability in chest compression rates for manual CPR.

Retrospective case-control ROSC upon arrival at study with matched Emergency Department control subjects. (Asytole/ Agonal, PEA and VTNF).

ROSC for case matched subjects in all rhythms (mechanical:

Mechanical CPR may improve ROSC and benefit patients with $39 \%$ versus manual: $29 \%, p=0.003$ ), non-shockable rhythms.

Asytole/agonal (mechanical: 37\%

vs. manual: $22 \%, p=0.008$ ), $P E A$ 4 minutes) and difficulty in

hard to identify potential benefits

of mechanical CPR. 
Use of an Automated Load-distributing Band Chest Compression Device for Out-of-Hospital Cardiac Arrest Resuscitation [12]

Adult patients over 18 years of age with nontraumatic OHCA.

Manual: 499

Mechanical: 210

7 Manual Chest Compression vs. Adult patients over Use of Automated chest 18 years of age compression device during non-traumatic OHCA resuscitation following out of and resuscitation hospital cardiac arrest: a attempted by EMS. randomized Manual: 517

Mechanical: 554

8 Clinical consequences of the introduction of chest compression in the EMS system for treatment of out-of-hospital cardiac arrest - a pilot study [24]

9 Mechanical active cardiopulmonary resuscitation non-traumatic OHCA (ACD-CPR) versus manual CPR Manual: 62 according to pressure of end tidal carbon dioxide Mechanical: 64 $\left(\mathrm{PET}_{\mathrm{ET}} \mathrm{CO}_{2}\right)$ during $\mathrm{CPR}$ in

out-of-hospital cardiac arrest (OHCA) [20]

10 Effectiveness of mechanical versus manual chest compressions in out-

of-hospital cardiac arrest resuscitation: a pilot study [2

Adult patients over 16 years of age with non-traumatic OHCA. Manual: 10 Mechanical:10

Adult patients over 18 years of age, witnessed non-traumatic OHCA.

Manual: 169

Mechanical: 159
All mechanical CPR subject

groups received manual

CPR prior to deployment

of the device.

AutoPulse was used.

Phased, non-randomized observational study of clinical outcomes of patients treated before and after transition from manual CPR to using mechanical CPR device,

AutoPulse.

Multi-centre randomized trial.

AutoPulse was used

Survival with spontaneous circulation 4 hours after emergency call is made,

Survival to hospital discharge, CPC at hospital discharge. pseudo-randomized trial. LUCAS was used.

Non-randomized, controlled ROSC at any time during treatment, Survival at hospita admission, survival to hospital discharge with neurological recovery

$\mathrm{ETCO}_{2}$ was measured after tracheal intubation for 15 minutes or until detection of ROSC. Readings were categorized to initial, minimum, average and maximum randomization study.

Odd days were using

$\mathrm{TCO}_{2}$ measurements at 5 minutes after intubation and CPR began, and a mechanical CPR and even second measure at initiation days using manual CPR. of transport to hospital.

Thumper was used.
$23 \%, p=0.079)$ and $V F N T$

(no difference).

ROSC (mechanical: 34.5\% vs manual: Mechanical CPR is better.

$20.2 \%$ ), Survival to hospital admission

(mechanical: 20.9\% vs. manual: 11.1\%),

Survival to hospital discharge

(mechanical: 9.7\% vs. manual: 2.7\%).

No difference in CPC $(p=0.360)$ and

OPC $(p=0.400)$ Better mechanical CPR

performance if ambulance response

time is less than 8 minutes.

No difference in survival to 4 hours (mechanical: $29.5 \%$ vs. manual: $28.5 \%, p=0.740$ ), survival to

Study was terminated when it was found that mechanical CPR was associated with worse discharge (mechanical. $5.8 \%$ vs. manual: 9.9\%, $p=0.060$ ). Difference in CPC score at hospital discharge (mechanical: $3.1 \%$ vs. manual $7.5 \%, p=0.006$ )

No significant difference in ROSC survival to hospital admission or hospital discharge.

No difference in CPC scores between 2 groups (majority 1 and 2).

$\mathrm{ETCO}_{2}$ was significantly higher in mechanical CPR as compared to manual CPR according to initia ( 3.38 vs. $2.71, p=0.010$ ), average ( 3.26 vs.2.69, $p=0.040$ ) and minimum (2.24 vs. $1.69, p=0.010$ ). No significant differences according to maximum value between 2 groups (4.88 vs.4.48, $p=0.230)$.No differences in survival outcomes and there was a long time interval between cardiac arrest to start of CPR and to ROSC for both groups.

Statistical significant between both groups. $80 \%$ in manual group has decreasing $\mathrm{ETCO}_{2}$ neurological outcomes and a trend towards worse survival than manual CPR.

No sufficient evidence to support that Mechanical CPR would improve outcome. This was due to long delay between arrival of ALS vehicle and use of Mechanical CPR (6 minutes).

Mechanical CPR performed compressions with higher cardiac output than manual chest compressions. while 20\% has increasing ETCO

Mechanical CPR produced better cardiac output overall, when compared to manual CPR.

All patients ( 3 excluded) in mechanical CPR do not have decreasing $\mathrm{ETCO}_{2}$. No difference in patient outcomes. 
When considering CPR quality, as defined by current AHA guidelines, results mostly favored mechanical CPR devices. The compression rate and depth was closer to established guidelines utilizing mechanical CPR devices as compared to CPR performed by rescuers. These factors held true through a variety of transport conditions and vehicle types as well. In addition, the compression ratio (number of compressions per minute) was closer to guideline recommendations when mechanical CPR was employed. In the one negative study regarding CPR quality, Wang [25] points out that most of the no-chestcompression intervals as it relates to mechanical CPR were related more to human error and that the compression rate was inadequate because the manufacturer's default rate was set at 5 compressions to every 1 breath. In addition, although the manual chest compression group in his study had higher instantaneous compression rates, the variability was also higher than that of mechanical CPR group and once the mechanical device was deployed, the instantaneous variability for the mechanical group was lower than the manual CPR group. From this, based upon the studies that were reviewed, mechanical CPR would appear to provide advantages over manual CPR as it relates to instantaneous and continuous CPR fractions throughout all phases of the resuscitation efforts. In addition, mechanical CPR devices appear to provide more consistently appropriate depths of compressions during transport of OHCA patients. However, there is difficulty generalizing the results found here on CPR quality due to small sample sizes, the retrospective nature of one of the studies, and because two of the studies are based upon mannequin evaluations, not real patients.

Clinical outcome measures in OHCA studies often include ROSC, survival to admission, 24-hour survival, and/or survival to discharge. Included in some studies is the CPC score as well as the OPC score. Both of these scores reflect the patient's ability to function and carry out activities of daily living.

There were six manuscripts that evaluated clinical outcomes in this review. The design of each study was slightly different, making generalizations about clinical outcomes more challenging. In Casner's [23] and Ong's [12] manuscripts, there were higher rates of ROSC, with Casner indicating that ROSC was achieved more successfully in asystole. However, the results of Casner's study have limited validity as late deployment of mechanical CPR devices by responders confounded the evaluation of ROSC because standard CPR was applied before the arrival of device (average 15 minutes). Ong's [12] larger prospective study demonstrated higher rates of ROSC and survival but no differences in neurological outcomes at discharge. Both of these studies reinforce the importance of consistent high quality and continuous CPR.
Hallstrom's [13] study was the only randomized controlled trial in this review, but found differing results than Ong's. A slightly larger study than Ong's but using the same device, Hallstrom chose to look at ROSC 4 hours after hospital admission. The study was stopped by the safety monitoring committee because of trends toward lower survival rates and poorer outcomes in mechanical CPR patients. Hallstrom's study design placed his intervention group into several different clusters, which varied the initial resuscitation efforts cluster to cluster. This heterogeneity between clusters makes Hallstrom's results more difficult to interpret. For example, one of the clusters focused on high quality, early, consistent CPR before the deployment of the Load Distributing Band (LDB) and before any analysis of the rhythm. As a subgroup, this particular cluster trended toward higher rates of ROSC.

Within the past several weeks, and after the preparation of this paper, the Circulation Improving Resuscitation Care (CIRC) trial [27] investigators presented their findings at the 2011 AHA Scientific Sessions in Orlando, Florida in November 2011. This prospective randomized trial of OHCA evaluated mechanical CPR using the LDB compared to "high-quality" manual CPR. The goal of the CIRC trial was to establish equivalence or superiority of one approach over the other. After enrolling over 4000 patients at five sites, and after adjusting for enrolment site, age, witnessed arrest and initial cardiac rhythm, the CIRC investigators found no difference in ROSC 24hour survival, survival to hospital discharge, and handsoff fraction when comparing mechanical CPR to manual CPR.

Until more evidence is available, it appears that outcomes may not be different between manual and mechanical CPR. We believe that the aim of any pre-hospital service should be to provide the optimum quality of CPR within the constraints and limitations of their operating environment. High quality manual CPR requires a commitment to training, quality review and adequate numbers of staff to attend to cardiac arrests. Mechanical CPR might possibly be helpful as a labor-saving device, but also requires a commitment to training and attention to deployment practices. It might conceivably be helpful from a crew safety viewpoint, reducing likelihood of injuries to crew performing CPR in a moving ambulance. The decision on which to choose would thus depend on the situation of each EMS service.

\section{Conclusion}

In this review, we found insufficient evidence to support or refute the use of mechanical CPR devices in settings of out-of-hospital cardiac arrest and during ambulance transport. While there is some low quality evidence suggesting that mechanical CPR can improve consistency 
and reduce interruptions in chest compressions, there is no evidence that mechanical CPR devices improve survival, to the contrary they may worsen neurological outcome.

\section{Abbreviations}

AHA: American heart association; CIRC: Circulation improving resuscitation Care; CPC: Cerebral performance categories; CPR: Cardio-pulmonary resuscitation; $\mathrm{ETCO}_{2}$ : End-tidal $\mathrm{CO}_{2}$; ILCOR: International liaison committee on resuscitation; LOE: Level of evidence; LDB: Load distributing band; OHCA: Out-of-hospital cardiac arrest; OPC: Overall performance categories; PAROS: Pan asian resuscitation outcomes study; PEA: Pulseless electrical activity; ROSC: Return of spontaneous circulation; VF: Ventricular fibrillation; VT: Ventricular tachycardia.

\section{Competing interests}

For this review paper, it includes information on resuscitation questions developed through the C2010 Consensus on Science and Treatment process, managed by the International Liaison Committee on Resuscitation (http:// www.amercian heart.org/ILCOR). The questions were developed by ILCOR Task Forces, using strict conflict of interest guidelines. In general, each question was assigned to two experts to complete a detailed structured review of the literature, and complete a detailed worksheet. Worksheets are discussed at ILCOR meetings to reach consensus and will be published in 2010 as the Consensus on Science and Treatment Recommendations (CoSTR). The conclusions published in the final CoSTR consensus document may differ from the conclusions in this review because the CoSTR consensus will reflect input from other worksheet authors and discussants at the conference, and will take into consideration implementation and feasibility issues as well as new relevant research.

Furthermore, MEH Ong receives grant support from Zoll Medical as principal investigator on several trials associated with load distributing band (LDB) CPR, Autopulse. The names of the trials are as follows:

(1) A Multicenter Randomised Controlled Trial Comparing Shock Success With Synchronized Defibrillation (Compression Upstroke Versus Precompression) During Ongoing Mechanical Cardiopulmonary Resuscitation In The Emergency Department

(2) Improving Quality of Cardiopulmonary Resuscitation in the Emergency Department with the Load Distributing Band Device Using Cardiac Arrest Teams and Video Assisted Quality Assurance. A phased non randomised study using historical controls

(3) Comparison Of Load Distributing Band And Standard Cardiopulmonary Resuscitation In Patients Presenting With Cardiac Arrest To Emergency Department

\section{Author details}

${ }^{1}$ Department of Emergency Medicine, Singapore General Hospital, Outram Road, Singapore 169608, Singapore. ${ }^{2}$ Department of Emergency Medicine, Kaiser Permanente, Sacramento, CA, USA. 'Department of Emergency System, Graduate School of Sport System, Kokushikan University, Tokyo, Japan. ${ }^{4}$ Department of Emergency Medicine, National Taiwan University, Taipei, Taiwan. ${ }^{5}$ Department of Emergency Medicine, William Beaumont Hospital, Royal Oak, MI, USA. ${ }^{6}$ Department of Emergency Medicine, Seoul National University Hospital, Seoul, Korea.

\section{Authors' contributions}

$\mathrm{MEOH}$ and KM conducted the systematic review. ZCZ assisted in the literature search and data extraction. All authors reviewed the data, contributed to and approved the final manuscript.

Received: 10 November 2011 Accepted: 3 June 2012 Published: 18 June 2012

\section{References}

1. Youngquist ST, Kaji AH, Niemann JT: Beta-blocker use and the changing epidemiology of out-of-hospital cardiac arrest rhythms. Resuscitation 2008, 76:376-380

2. Bunch TJ, White RD: Trends in treated ventricular fibrillation in out-ofhospital cardiac arrest: Ischemic compared to non-ischemic heart disease. Resuscitation 2005, 67:51-54.
3. Hess EP, Campbell RL, White RD: Epidemiology, trends, and outcome of out-of-hospital cardiac arrest of non-cardiac origin. Resuscitation 2007, 72:200-206.

4. Polentini MS, Pirrallo RG, McGill W: The changing incidence of ventricular fibrillation in Milwaukee, Wisconsin (1992-2002). Prehosp Emerg Care 2006, 10:52-60.

5. Rea TD, Pearce RM, Raghunathan TE, Lemaitre RN, Sotoodehnia N, Jouven X, Siscovick DS: Incidence of Out-of-Hospital cardiac arrest. Am J Cardiol 2004, 93:1455-1460.

6. Nichol G, Thomas E, Callaway CW, Hedges J, Powell JL, Aufderheide TP, Rea T, Lowe R, Brown T, Dreyer J, Davis D, Idris A, Stiell I: Regional Variation in Out-of-Hospital Cardiac Arrest Incidence and Outcome. JAMA 2008, 300:1423-1431.

7. Wik L, Kramer-Johansen J, Myklebust H, Sorebo H, Svensson L, Fellows B, Steen PA: Quality of Cardiopulmonary Resuscitation During Out-ofHospital Cardiac Arrest. JAMA 2005, 293:299-304.

8. Kern KB: Coronary perfusion pressure during cardio-pulmonary resuscitation. Best Pract Res Clin Anaesthesiol 2000, 14:591-609.

9. Cobb LA, Fahrenbruch CE, Walsh TR, Copass MK, Olsufka M, Breskin M, Hallstrom AP: Influence of Cardiopulmonary Resuscitation Prior to Defibrillation in Patients With Out-of-Hospital Ventricular Fibrillation. JAMA 1999, 281:1182-1188.

10. Wik L: Rediscovering the importance of chest compressions to improve the outcome from cardiac arrest. Resuscitation 2003, 58:267-269.

11. Wik L, Hansen TB, Fylling F, Steen Tr, Vaagenes P, Auestad BrH, Steen PA: Delaying Defibrillation to Give Basic Cardiopulmonary Resuscitation to Patients With Out-of-Hospital Ventricular Fibrillation. JAMA 2003, 289:1389-1395.

12. Ong MEH, Ornato JP, Edwards DP, Dhindsa HS, Best AM, Ines CS, Hickey $S$, Clark B, Williams DC, Powell RG, Overton JL, Peberdy MA: Use of an Automated, Load-Distributing Band Chest Compression Device for Outof-Hospital Cardiac Arrest Resuscitation. JAMA 2006, 295:2629-2637.

13. Hallstrom A, Rea TD, Sayre MR, Christenson J, Anton AR, Mosesso VN, Van Ottingham L, Olsufka M, Pennington S, White L, Yahn S, Husar J, Morris MF, Cobb LA: Manual Chest Compression vs Use of an Automated Chest Compression Device During Resuscitation Following Out-of-Hospital Cardiac Arrest. JAMA 2006, 295:2620-2628.

14. Morley PT, Atkins DL, Billi JE, Bossaert L, Callaway CW, de Caen AR, Deakin CD, Eigel B, Hazinski MF, Hickey RW, Jacobs I, Kleinman ME, Koster RW, Mancini ME, Montgomery WH, Morrison L, Nadkarni VM, Nolan JP, O'Connor RE, Perlman JM, Sayre MR, Semenko TI, Shuster M, Soar J, Wyllie J, Zideman D: Part 3: evidence evaluation process: 2010 International Consensus on Cardiopulmonary Resuscitation and Emergency Cardiovascular Care Science With Treatment Recommendations. Circulation 2010, 122 (16 Suppl 2):S283-290.

15. Richardson WS, Wilson MC, Nishikawa J, Hayward RS: The well-built clinical question: a key to evidence-based decisions. ACP J Club 1995, 123:A12-A13.

16. Brooks SC, Bigham BL, Morrison L: Mechanical versus manual chest compressions for cardiac arrest. Cochrane Database Sys Rev 2011, 1: CD007260.

17. Lim SH, Shuster M, Deakin CD, Kleinman ME, Koster RW, Morrison L, Nolan JP, Sayre MR: Part 7: CPR techniques and devices: 2010 International Consensus on Cardiopulmonary Resuscitation and Emergency Cardiovascular Care Science with Treatment Recommendations. Resuscitation 2010, 81(1, Supplement):e86-e92.

18. Stapleton ER: Comparing CPR during ambulance transport. Manual vs. mechanical methods. J Emerg Med Serv JEMS 1991, 16:63-64. 66, 68 passim.

19. Sunde K, Wik L, Steen PA: Quality of mechanical, manual standard and active compression-decompression CPR on the arrest site and during transport in a manikin model. Resuscitation 1997, 34:235-242.

20. Axelsson C, Karlsson T, Axelsson AB, Herlitz J: Mechanical active compression-decompression cardiopulmonary resuscitation (ACD-CPR) versus manual $C P R$ according to pressure of end tidal carbon dioxide $\left(\mathrm{P}_{\mathrm{ET}} \mathrm{CO}_{2}\right)$ during CPR in out-of-hospital cardiac arrest (OHCA). Resuscitation 2009, 80:1099-1103.

21. Dickinson ET, Verdile VP, Schneider RM, Salluzzo RF: Effectiveness of mechanical versus manual chest compressions in out-of- hospital cardiac arrest resuscitation: A pilot study. Am J Emerg Med 1998, 16:289-292.

22. Olasveengen TM, Wik L, Steen PA: Quality of cardiopulmonary resuscitation before and during transport in out-of-hospital cardiac arrest. Resuscitation 2008, 76:185-190. 
23. Casner M, Andersen D, Isaacs SM: The impact of a new CPR assist device on rate of return of spontaneous circulation in out-of-hospital cardiac arrest. Prehosp Emerg Care 2005, 9:61-67.

24. Axelsson C, Nestin J, Svensson L, Axelsson AB, Herlitz J: Clinical consequences of the introduction of mechanical chest compression in the EMS system for treatment of out-of-hospital cardiac arrest-A pilot study. Resuscitation 2006, 71:47-55.

25. Wang $\mathrm{HC}$, Chiang WC, Chen SY, Ke YL, Chi CL, Yang CW, Lin PC, Ko PCl, Wang YC, Tsai TC, Huang CH, Hsiung KH, Ma MHM, Chen SC, Chen WJ, Lin FY: Video-recording and time-motion analyses of manual versus mechanical cardiopulmonary resuscitation during ambulance transport. Resuscitation 2007, 74:453-460.

26. Russi CS KL, Myers LA, Hess EP, White RD: A Comparison of Chest Compression Quality Delivered During On-Scene and Transport Cardiopulmonary Resuscitation. Prehosp Emerg Care 2011, 15(1):106.

27. Wik L, Olsen J, Persse D, Sterez F, Lozano M, Brouwer MA, Westfall M, Souders CM, Malzer R, van Grunsven PM, Travis D, Whitehead A, Herken UR, Lerner E: Comparison of Survival to Hospital Discharge between Integrated AutoPulse-CPR and Manual-CPR during out-of-hospital cardiac arrest of presumed cardiac orgin: The Circulation Improving Resuscitation Care (CIRC) Trial. In Proceedings of Amercian Heart Association Scientific Session:; 2011. 12-16 November 2011, in press.

doi:10.1186/1757-7241-20-39

Cite this article as: Ong et al:: Mechanical CPR devices compared to manual CPR during out-of-hospital cardiac arrest and ambulance transport: a systematic review. Scandinavian Journal of Trauma, Resuscitation and Emergency Medicine 2012 20:39.

\section{Submit your next manuscript to BioMed Central and take full advantage of:}

- Convenient online submission

- Thorough peer review

- No space constraints or color figure charges

- Immediate publication on acceptance

- Inclusion in PubMed, CAS, Scopus and Google Scholar

- Research which is freely available for redistribution 